\title{
Lineamientos para el diseño de interfaces gráficas y componentes gráficos de sistemas de comunicación aumentativa y alternativa
}

\section{Resumen}

Las personas con dificultades en la producción del lenguaje deben enfrentarse a situaciones que les hace depender día a día de otras personas, ya que no pueden comunicar sus pensamientos ni necesidades. Para ayudar a estas personas los sistemas de comunicación aumentativa y alternativa (CAA) ofrecen la posibilidad de transmitir información a partir de la selección de componentes gráficos que representan la realidad, entre los problemas de este tipo de sistemas se encuentran los bajos niveles de usabilidad en las interfaces gráficas y el uso de pictogramas que no se adecuan al contexto y lenguaje de los usuarios. Este trabajo presenta una serie de factores a tener en cuenta al momento de diseñar una interfaz gráfica para sistemas CAA y la evaluación de material gráfico diseñado específicamente para el contexto de los usuarios con afasia, una enfermedad que se caracteriza por la pérdida de la capacidad de producir y comprender el lenguaje oral y escrito. Las pruebas señalan que adicional a la posibilidad de modificar factores de posición, tamaño y color, es necesario incluir imágenes que hagan parte del contexto del usuario facilitando la selección de las imágenes relevantes para la creación del mensaje. Los resultados de esta propuesta pueden ser de utilidad para desarrolladores de sistemas CAA ya que sirven como guía para el diseño de interfaces que facilitan al usuario la creación de frases.

\author{
Cristian Andrés Narváez \\ Alarcón \\ Maestría en Ingeniería de Sistemas y \\ Computación \\ Universidad Militar Nueva Granada. \\ Bogotá, Colombia \\ Correo electrónico: cristian. \\ narvaezing@gmail.com \\ 능 orcid.org/0000-0002-0059-5441 \\ Google Scholar
Germán Andrés Garnica
Gaitán
Maestría en Diseño Industrial
Universidad Militar Nueva Granada.
Correo electrónico: german.garnica@ unimilitar.edu.co
ำ orcid.org/0000-0002-8398-6404
Google Scholar \\ Bogotá, Colombia
}

\section{Recibido: Abril 9 de 2018} Aprobado: Mayo 30 de 2019

\section{Palabras clave:}

Diseño de interfaces gráficas, sistema de comunicación aumentativa y alternativa (CAA), usabilidad. 


\section{Graphic interfaces and graphic components design guidelines for Augmentative and Alternative Communication Systems}

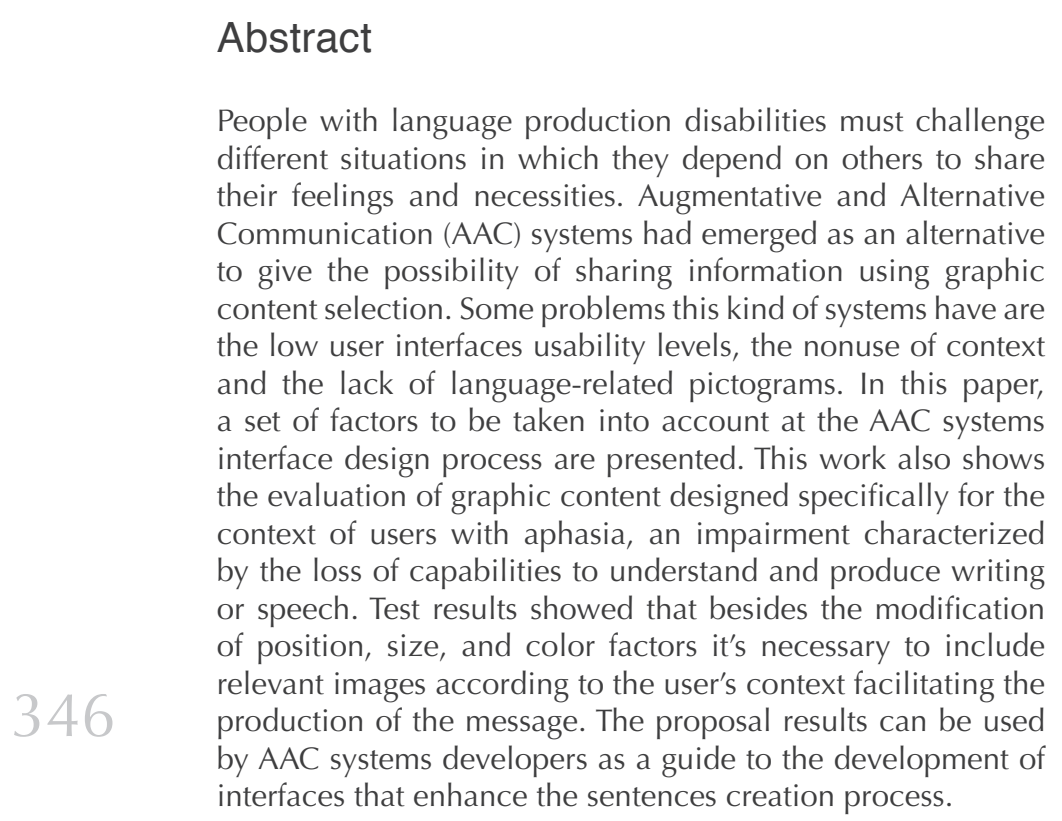

Key words:

Augmentative and alternative communication systems (AAC), graphic interfaces design, usability. 


\section{Introducción}

Las personas con dificultades en la producción del lenguaje y la comunicación tienen la capacidad de comprender lo que otras personas quieren comunicarles, pero no son capaces de expresar sus pensamientos y necesidades, construir relaciones, tomar decisiones en contextos sociales o incluso pedir información a desconocidos. Lo anterior se da por factores como la falta de control de los órganos del habla o daños cognitivos que se pueden dar desde el nacimiento o pueden desarrollarse en diferentes etapas de la vida producto de accidentes cerebrovasculares u otros. Enfermedades neurológicas como el síndrome de Down, autismo, afasia, se caracterizan por afectar en gran medida la calidad de vida de las personas (Lam \& Wodchis, 2010)., y dependiendo del grado de afectación de las habilidades comunicativas, es posible el desarrollo de dependencia con los individuos más cercanos como aquellos que les cuidan. Teniendo en cuenta la afectación en la calidad de vida de las personas por la inhabilidad de comunicar, las necesidades de comunicación complejas (NCC) se agrupan en tres categorías principales: (i) aquellos que no pueden producir el lenguaje y se apoyan en otras formas de comunicación como los gestos; (ii) aquellos que pueden producir el lenguaje, pero no es sencillo comprender lo que quieren decir; y (iii) aquellos que pueden producir el lenguaje, pero no pueden entender lo que otros dicen (The State of Queensland, 2018).

La afasia es una de las enfermedades que se caracterizan porque los pacientes pierden la capacidad de producir y comprender el lenguaje y existe la posibilidad de que estos pierdan sus capacidades motoras (Hillis, 2007). Esta enfermedad se puede dar por diferentes razones, pero la más común son los accidentes cerebrovasculares. Adicionalmente, esta enfermedad es de especial interés porque entre el grupo de enfermedades neurológicas es la que más afecta el nivel de vida de quienes la padecen (Lam \& Wodchis, 2010)., ya que dentro de sus características se puede encontrar, según el tipo y nivel 
de afectación, la repetición de frases, la poca comprensión de lo que se les dice, producción espontánea y sin fluidez de frases, límites en el número de palabras que conocen y confusión entre lo que piensan que dicen y lo que realmente están diciendo (Hillis, 2007). Las personas con afasia suelen aislarse en su propio entorno dejando de lado sus relaciones de amistad y abandonando las actividades sociales por miedo al rechazo, lo cual dificulta el proceso de aceptación y recuperación de la enfermedad, y puede llevar a la depresión en el paciente. También es posible que las personas eviten el contacto con la persona dada la dificultad en la comunicación (Davidson, Howe, Worral, Hickson \& Togher, 2008). Para desenvolverse en situaciones sociales, las personas con NCC deben recurrir a métodos diferentes al habla o la escritura como lo son el lenguaje de señas, los gestos, la referencia a objetos de la realidad y los sistemas computacionales. Por lo anterior, la comunicación aumentativa y alternativa (CAA) estudia y resuelve los desórdenes presentes en la comunicación del ser humano y la forma en la que las habilidades de comunicación se pueden recuperar. A tal efecto, se usan símbolos y otras estrategias que permiten comunicar a aquellos que no pueden producir el lenguaje. Una de las principales metas de un sistema CAA es que este pueda ser usado día a día en diferentes contextos, ayudando a solucionar los retos que enfrentan las personas con NCC al momento de comunicar (Light \& McNaughton, 2012; Chan, 2014; Belani, 2012).

Los sistemas CAA enfrentan diferentes retos en la actualidad:

1. Si se da en dispositivos móviles, estos cuentan con diversidad de gestos que son difíciles de ejecutar para personas con inhabilidades motoras e incluyen pocos mecanismos para ayudar a los usuarios a aprender y ejecutar los mismos.

2. El desarrollo rápido y cambio constante de las aplicaciones puede ser problemático para individuos a los cuales se les dificulta adaptarse al cambio. 
3. Un solo diseño no es funcional para todas las personas con NCC.

4. El uso de interfaces muy dinámicas exige revisar la usabilidad de la interfaz para el común de los casos y elección de cada individuo.

5. La aplicación debe maximizar la comunicación y la participación de las personas en contextos sociales (Light \& McNaughton, 2012; Stent \& Bangalore, 2014).

Para solucionar algunos de estos retos (1, 3 y 4) existe la necesidad de trabajar sobre los conceptos de usabilidad en los sistemas CAA; según la Organización Internacional para la Estandarización, la usabilidad se define como "el grado en que un producto puede ser usado por un tipo de usuarios en específico para cumplir metas en específico con efectividad, eficiencia y satisfacción en un contexto específico de uso" (International Organization for Standardization [ISO], 2018) En una revisión extensa de la literatura, Seffah (Seffah, Donyaee, Kline \& Padda, 2006)., identifica 12 factores importantes en la medición de la usabilidad: eficiencia, efectividad, productividad, satisfacción, facilidad de aprendizaje, seguridad, confianza, accesibilidad, globalidad, utilidad, portabilidad y adaptabilidad. Relacionado a lo anterior, Saturno (Saturno, Ramirez, Conte, Farhat \& Piucco, 2015)., propone la creación de una herramienta CAA, basado en los tableros de comunicación e iconos iconográficos, que le permite a los usuarios seleccionar símbolos que pueden ser personalizados y que son recomendados según las necesidades del usuario. Así mismo, en este artículo se consideran diferentes características de usabilidad teniendo en cuenta las necesidades del usuario y las recomendaciones de las guías de accesibilidad del contenido a la web. Al-Arifi (Al-Arifi, Al-Rubaian, Al-Ofisan, Al-Romi \& Al-Wabil, 2013)., presenta una herramienta CAA para sistemas iOS que funciona en el lenguaje arábigo, que es diseñado para ser usado por personas con autismo en contextos educativos, y que tuvo en cuenta a los usuarios finales en el proceso de desarrollo. En esta propuesta se le presentan al usuario las imágenes en categorías, el usuario puede seleccionar una 
lista de favoritos y registrar respuestas rápidas para continuar con el proceso de comunicación. Keskinen (Keskinen, Heimonen, Turunen, Rajaniemi \& Kauppinen, 2012)., presenta SymbolChat un sistema de comunicación desarrollado con la ayuda de profesionales de diferentes áreas, con lo cual se tienen en cuenta las necesidades de los usuarios desde diferentes perspectivas, también tiene en cuenta la necesidad de personalización de los elementos que se muestran al usuario. Uno de los aspectos más relevantes de esta propuesta es que los iconos presentados son los mismos que contiene el sistema DynaVox por lo cual las personas que interactúan con el sistema no deben aprender a identificar y relacionar un nuevo conjunto de símbolos. Mahmud (Mahmud \& Martens, 2016)., presenta un sistema de comunicación que le permite a las personas con afasia, cuidadores y familiares crear mensajes que son enviados por medio de un correo electrónico. La herramienta permite agregar nombres y fotos de amigos los cuales puede registrar el usuario o encontrarlos según su ubicación. Esta herramienta también tiene soporte de lenguaje, el cual presenta al usuario palabras que se relacionan con una temática específica y que les facilita la escritura de los mensajes. Aunque el propósito de esta herramienta no es comunicar en cualquier contexto y tiempo real, les permite a estos comunicar acciones desarrolladas durante el día o deseos. Hornero (Hornero, Conde, Quílez, Domingo, Rodriguez, Romero \& Casas, 2015)., presenta en su trabajo un dispositivo de CAA que combina los tableros de comunicación y la naturalidad oral de los comunicadores electrónicos. Estos comunicadores se conectan por medio de una red inalámbrica, permitiendo que los tableros de comunicación puedan ser cambiados de acuerdo con las necesidades motoras y comunicativas del usuario, y que además, puedan ser reemplazados algunos componentes por dispositivos con los que ya cuente el usuario. Adicional a las propuestas anteriores existen sistemas CAA comerciales como DynaVox [1], que llevan varios años en el mercado y se han adaptado a los avances tecnológicos para brindar mejores soluciones a los usuarios. 
Aunque existen diferentes propuestas en el estado del arte algunas de estas tienen un diseño de interfaz gráfica y formas de interacción que no toman en cuenta las habilidades de los usuarios objetivo y que representan para estos una barrera para el uso de la tecnología (Mahmud \& Martens, 2016). Tampoco se incluye dentro del proceso de diseño de interacción un método que permita la selección rápida de los términos que compondrán la frase (Hornero et al., 2015)., y en términos generales no se mide la usabilidad de los diferentes componentes que se presentan al usuario (Saturno et al., 2015). Este problema ha aumentado dada a la adopción de los sistemas CAA en la industria, puesto que diferentes grupos liberan sistemas que no cumplen con las necesidades de los usuarios y que no tienen un trasfondo de investigación y desarrollo (Light \& McNaughton, 2012).

Teniendo en cuenta lo anterior, es necesario desarrollar una herramienta de comunicación que tenga en cuenta las necesidades y habilidades de las personas con afasia y que se centre en mejorar la usabilidad del sistema.

En este trabajo se presentan pautas que se deben seguir para el diseño de una interfaz centrada en el usuario para sistemas de comunicación aumentativa y alternativa, inspirados en los tableros de comunicación y los símbolos iconográficos, así como el diseño y evaluación de una serie de íconos que buscan facilitar etapas de entendimiento y relación de personas que hablan el idioma español y que se encuentran en la ciudad de Bogotá, Colombia. El resto de este artículo está organizado de la siguiente manera: en la sección II, se definen algunos de los retos de los sistemas CAA y se introduce al lector en el proyecto relator. En la sección III, se presentan las pruebas para validar los pictogramas propuestos y las características de la población de la prueba. La sección IV incluye los resultados relevantes obtenidos de las pruebas y la discusión de la información encontrada. Finalmente, en la sección $\mathrm{V}$ se presentan las conclusiones y el trabajo futuro de esta propuesta. 


\section{Retos de los sistemas CAA y Proyecto Relator}

En la sección I se nombran algunas problemáticas que deben ser solucionadas en los sistemas CAA sin embargo, existen características identificadas en el estado del arte (Mahmud \& Martens, 2016)., que se relacionan con la forma en la que el usuario usa los sistemas de comunicación como: (i) los pacientes con afasia usan pocas categorías para seleccionar los componentes del mensaje, por lo tanto, es necesario ordenar el contenido de forma tal que, se facilite la selección al usuario; (ii) es difícil encontrar iconos que permitan a las personas comunicar lo deseado, por lo tanto, es necesario crear una base de diseños gráficos que tengan en cuenta el contexto del usuario; (iii) los mensajes que produce un paciente con afasia son generalmente cortos; (iv) si no se tiene en cuenta la usabilidad en el sistema es posible que el usuario abandone el mismo; y (v) se debe ofrecer un soporte de lenguaje al usuario para que este pueda crear el contenido del mensaje. En el estudio realizado por Salminen (Salminen, Petrie \& Ryan, 2004) ., se identifica que después de un periodo de tres a seis meses los participantes pierden la motivación necesaria para emplear el sistema CAA, dados los bajos niveles de usabilidad y que ninguno de los participantes tomó la herramienta digital de CAA como su método de comunicación. Lo anterior es producto de la difícil configuración del sistema, el limitado vocabulario disponible y la lentitud que sobrelleva el proceso de comunicación. Sin embargo, también señala que este tipo de sistemas logra mejorar las habilidades del habla brindando una forma de comunicación independiente, precisa y autónoma. Adicionalmente, el uso de los sistemas no representa riesgos para el desarrollo y recuperación del lenguaje, no existen prerrequisitos cognitivos para el uso de éstos y el uso de este tipo de sistemas es beneficioso para niños y adultos (Light \& McNaughton, 2012).

Los problemas de los sistemas de CAA han sido tratados en el proyecto Relator desde diferentes perspectivas. Relator es un sistema CAA perteneciente al 
grupo de asistencia con alto grado de componentes técnicos y tecnológicos (Chan, 2014). , que busca suplir las necesidades de la población con NCC en el contexto latinoamericano de Colombia, para esta etapa del proyecto, cuya población es hispanohablante. A partir de conversaciones con profesionales en fonoaudiología se identifica la necesidad de brindar soporte a las personas con afasia para que éste comunique las actividades que desarrolló durante el día. En un principio los esfuerzos se concentraron en la identificación de técnicas computacionales que permitieran facilitar al usuario la creación de frases coherentes, las ontologías, como una forma de representar el conocimiento de un dominio, fueron seleccionadas para aumentar la calidad semántica de los textos creados por el usuario (Mancilla, Sastoque, Mendoza \& Iregui, 2013). El tiempo de producción de mensajes es uno de los inconvenientes que tienen los sistemas de comunicación, y para solucionar esto se propuso un modelo de recomendación centrado en el usuario para sistemas CAA (Sastoque, Mancilla, Mendoza \& Iregui)., el cual sugiere y predice los componentes principales de una frase teniendo en cuenta los componentes de comunicación de preferencia para el usuario. Adicional al proceso de recomendación, se propone un método para la personalización de ontologías que permite a un usuario relacionar los componentes de las frases que desea generar con entidades de su vida cotidiana (Mancilla, Sastoque \& Iregui, 2015). Finalmente, la calidad sintáctica de los mensajes producidos por los usuarios fue tratada por medio de la propuesta de un motor de generación de lenguaje natural para el lenguaje español, el cual usa elementos provenientes del modelo de representación de conocimiento y reglas gramaticales propias del idioma (Narváez, Sastoque \& Iregui, 2015).

Las problemáticas asociadas al diseño de usabilidad e interacción en sistemas computacionales para personas con NCC, específicamente personas con afasia, son tratadas en este artículo teniendo en cuenta algunos componentes de la metodología propuesta por Garnica (Sastoque, Narváez \& Garnica, 2015). La participación del usuario es el eje fundamental de las actividades para la creación de interfaces gráficas centradas en el usuario. 
Un primer análisis de las características de usabilidad que permitieran facilitar el proceso de creación de frases en un sistema de comunicación, en donde los componentes de las frases son seleccionados por medio de elementos gráficos, se lleva a cabo a partir de la realización de pruebas en donde se tomó una población de 50 individuos de diferentes edades y género. Adicionalmente, Dumas (Dumas, 2002) expone que las pruebas de usabilidad se pueden dar con un mínimo de cuatro participantes y que un rango de 10 a 12 es suficiente para encontrar el $80 \%$ de las características de usabilidad de un sistema respaldando la cantidad de personas usadas en las pruebas. Esta población cuenta con una profesión y cumple con las capacidades de comunicación necesarias para desenvolverse en contextos sociales, lo cual aporta algunas condiciones con las que esperan los individuos se puedan comunicar, o se les comunique algo. Adicionalmente, algunos individuos cuentan con más de 60 años agregando al estudio los límites que representan similitudes en las capacidades motoras y de uso de tecnología de una persona con NCC. Producto de estas pruebas se obtiene que: (i) al usuario le interesa cambiar el orden y tamaño de los elementos a seleccionar en la interfaz, como las categorías que agrupan los conceptos a comunicar y los propios conceptos; (ii) los colores de los elementos de la interfaz gráfica deben contrastar entre sí, facilitando la identificación y selección de áreas de interacción; (iii) a los usuarios les interesa utilizar contenidos de su realidad, que les facilite la selección de los componentes en la interfaz; y finalmente (iv) otras consideraciones en cuanto a la disposición de los elementos de la interfaz. Desde lo anterior fue posible el diseño de la interfaz gráfica que se puede observar en la Figura 1. 


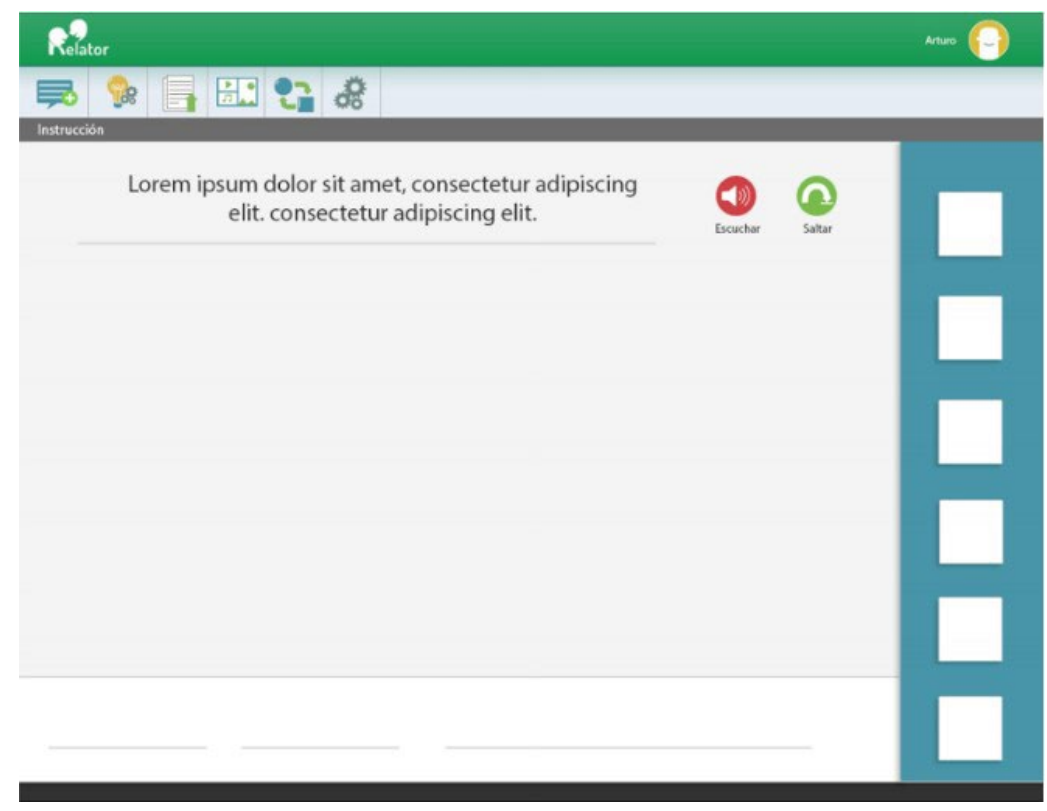

Figura 1. Ejemplo de algunas interfaces gráficas del sistema.

Las propuestas del estado del arte y el origen de los tableros de comunicación demuestran lo importantes que son las imágenes para que una persona con NCC seleccione y conecte conceptos para crear una frase. El conocimiento empírico de los profesionales en fonoaudiología señala que los contenidos visuales que no son diseñados teniendo en cuenta el lenguaje nativo del paciente, incluyendo los modismos de este, o el contexto de uso de objetos del mismo, no son los más adecuados para ser usados en terapias de rehabilitación o en procesos de comunicación. Es decir, se precisa de un grado de especificidad o personalización, aportando familiaridad al contenido visual. 
Blake (Blake, 2000)., indica que el uso de formas de comunicación simbólicas o no, depende de la cultura de cada individuo y que son sus experiencias las que limitan su comprensión sobre una imagen. Van (Van \& Alan, 2004)., mide cómo un set de imágenes establecidas generadas para un contexto general es asociado por personas de educación terciaria que cuentan con un contexto y una cultura específica, el estudio indica que es necesaria la personalización de los íconos para los usuarios de sistemas CAA en diferentes contextos culturales, también indica que es necesario tener en cuenta las expresiones verbales y recomienda la inclusión de los usuarios en el proceso de desarrollo de la aplicación.

Lo anterior llevó a la creación de 450 pictogramas que tienen en cuenta las expresiones verbales y el contexto cultural de los usuarios del sistema Relator, y que representan siete categorías estudiadas en el trabajo de Mancilla (Mancilla et al, 2013).: actividad, situación, lugar, transporte, motivo, persona y tiempo. Para lo anterior, un profesional en diseño gráfico e ilustración fue acompañado por un profesional en fonoaudiología (PF), quien lo asesoró en elementos visuales y conceptuales con el objetivo de facilitar a los usuarios la asociación de conceptos presentes en la interfaz gráfica del sistema. Entre las características de los pictogramas diseñados se encuentra el uso de colores planos y pasteles que contrastan entre sí, el uso de texto en la parte inferior del contenido con una letra legible y con un tamaño proporcional a las dimensiones del elemento visual, y finalmente el uso de un color único que identifica cada una de las siete categorías y del cual se hace uso en un marco que encierra el contenido del concepto y el texto de la ficha. Adicionalmente todos los pictogramas tienen un estilo gráfico determinado y compartido, lo que facilita al usuario el aprendizaje y uso de los mismos. En la figura 2 se pueden apreciar algunos pictogramas diseñados para el sistema Relator. La prueba realizada al grupo de pictogramas puede verse en la sección III. 


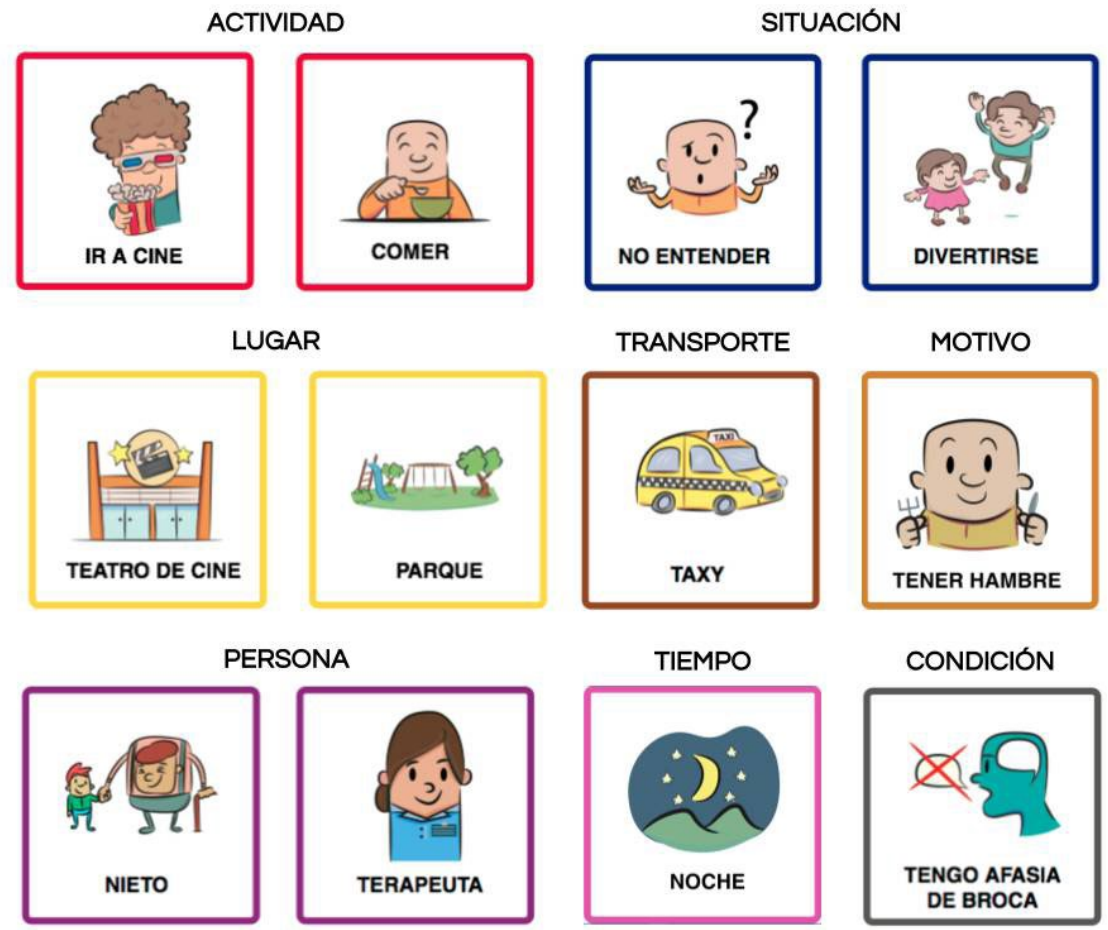

Figura 2. Pictogramas diseñados para el sistema CAA Relator, los colores identifican a cada una de las categorías.

\section{Pruebas}

Se realizaron pruebas de los iconos creados en Bogotá D.C., Colombia, ya que las personas que pertenecen a esa zona geográfica son el objetivo del presente sistema CAA. El primer objetivo de la realización de esta prueba es medir la pertinencia de la representación gráfica de un contenido específico y el tiempo de lectura e interpretación de dicho contenido, el segundo objetivo busca 
identificar aspectos relacionados con la facilidad de lectura, funcionalidad, nivel de error, satisfacción y especificidad del contenido de los pictogramas diseñados.

Se toma un conjunto de 21 pictogramas que cubren diferentes categorías en modo de representación de conceptos y que son relevantes para el contexto de las personas con NCC. Para esta prueba se tiene un grupo de participantes compuesto por cinco individuos con necesidades complejas de comunicación y 12 individuos sin limitación comunicativa y cognitiva. Para el primer grupo de individuos se eligen personas con un rango de edad de 20 a 40 años con una enfermedad desarrollada en un tiempo mayor a 3 años y sin contacto previo con herramientas de CAA. Para el segundo grupo de individuos se seleccionan participantes de 18 años o más, con y sin contacto con personas con NCC, y con o sin contacto con herramientas de tipo CAA. En cuanto a los participantes con necesidades complejas de comunicación se cuenta con dos mujeres, una mayor de 60 años y con dificultades de audición y memoria, presenta muy bajo uso de tecnología computacional; la otra mujer es menor de 30 años con sordera y alta dificultad de comunicación verbal, usa el lenguaje de señas y no tiene inconveniente de lectura y uso de tecnología. Dos hombres menores de 30 años presentan sordera y su comunicación es principalmente mediante lenguaje de señas, el tercer individuo de este grupo es un hombre mayor de 70 años con dificultades de memoria y escritura. Este grupo de personas con NCC, sin afasia, presentan condiciones pertinentes a un sistema CAA y no han tenido experiencia previa con sistemas similares, pero no presentan limitantes de capacidad de uso de tecnología computacional multimedia más allá de la condición particular de cada uno, como la percepción de sonidos, comunicación por voz, comunicación por señas y comunicación por lenguaje escrito.

La prueba está dividida en dos etapas: la primera etapa, E1, permite conocer las interpretaciones o lectura del contenido gráfico que se le puede dar a 
un pictograma, para lo anterior se presentan ocho dibujos como se puede observar en la figura 3. La segunda etapa, E2, tiene como objetivo conocer si el concepto contenido por el pictograma para el cual fue diseñado corresponde al que prefieren o entienden los usuarios desde su particular interpretación, para esta etapa se usan 11 imágenes que se asocian a diferentes conceptos y categorías (Fig. 3).

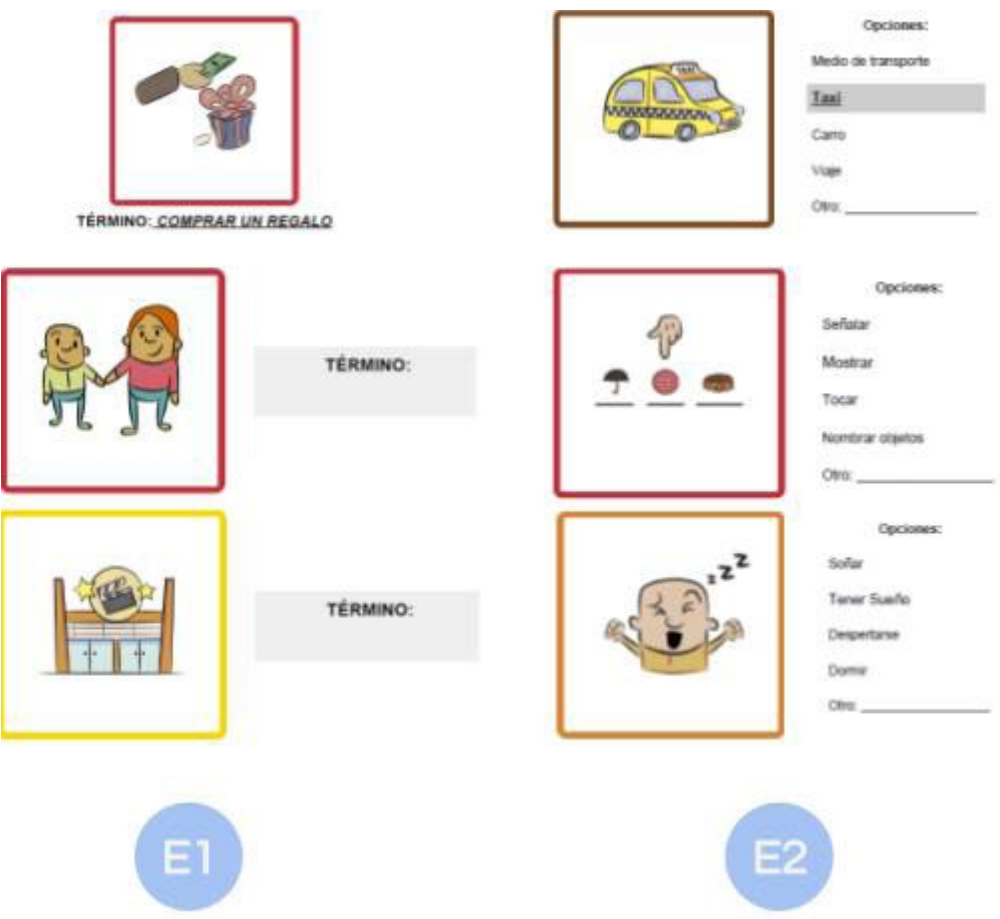

Figura 3. Contenidos de las pruebas realizadas para la validación de pictogramas. (E1) Ejercicio de descripción de concepto. (E2) Ejercicio de asociación. 


\section{Procedimiento para la realización de pruebas}

Para las pruebas E1 y E2 se da una sesión de 40 minutos en la cual participan cuatro posibles actores: (i) usuario A, persona con NCC; (ii) usuario B, persona sin ningún tipo de limitación comunicativa y cognitiva; (iii) moderador, profesional que posee habilidades para guiar las actividades de los usuarios A y B; (iv) observador, profesional encargado de registrar los datos comportamentales de los usuarios A y B. Dentro del tiempo de la ejecución se toman 5 a 10 minutos para explicar el propósito y dinámicas de la prueba, alrededor de 20 minutos para la ejecución de las actividades de la prueba, y los últimos minutos se disponen para que el usuario registre en formatos, que se han entregado anteriormente, opiniones y recomendaciones acerca de la prueba.

La prueba inicia cuando se hace entrega del material al usuario, en la primera etapa (E1), se le presentan al individuo una serie de pictogramas con el propósito de que este escriba las palabras que mejor describen el contenido de la imagen como se puede ver en la figura 3. Lo anterior permite conocer las interpretaciones que se le pueden dar a un pictograma y reconocer los tiempos de los individuos en su interpretación.

En la etapa 2 (E2), se realiza un ejercicio de asociación en donde se presenta una imagen y el usuario selecciona, entre una lista de términos, aquella palabra que él entiende representa el pictograma. Un ejemplo de esta prueba se puede ver en la figura 3.

\section{Resultados y Discusión}

Partiendo de los datos de la prueba, se evidencian algunas tendencias, orden e ideas finales. La figura 4 muestra el tiempo máximo y mínimo que se presentó entre los usuarios A y B para finalizar cada una de las etapas. Para el caso, uno 
de los individuos B realizó más actividades a las enunciadas para la prueba E1, tomándole 22 minutos con 21 segundos finalizar la misma, al ser este un dato atípico se excluyó del conteo de tiempo para la gráfica relacionada. Se observa que generalmente a los usuarios con NCC les toma más tiempo la realización de las actividades asociadas a cada una de las etapas. Adicionalmente, la realización de la E2, ejercicio de asociación, registró más tiempo para el general de los individuos. Lo anterior se puede entender dada la cantidad de conceptos y la naturaleza del ejercicio, pues los individuos tienden a comparar la información antes de seleccionar la respuesta. En promedio, el tiempo de lectura del contenido gráfico y significación frente a cada pictograma en E2 de la prueba fue de 1 minuto con 26 segundos mientras que para la E1 el tiempo fue de 1 minuto con 7 segundos. Al considerar la totalidad de individuos de tipo A y B se tiene que la interpretación del contenido específico y la lectura del mismo utilizando solo imágenes, se realiza en un tiempo promedio de 67 segundos y, utilizando imagen y texto — pictograma - el tiempo promedio fue de 86 segundos. Estos tiempos permiten tener un parámetro de comparación frente a las pruebas con sistemas CAA ya que se esperaría en lo posible no superar este margen.

Sabiendo que el contexto y experiencias de los individuos son importantes para la interpretación de los pictogramas, que se busca medir la condición comunicativa de las representaciones gráficas contenidas, y el tiempo de lectura para su uso; se ha considerado al momento de interpretar los datos que son: aciertos (A) cuando los usuarios coinciden con el concepto para el cual se diseñó el pictograma, no aciertos $(X)$ cuando el término es completamente diferente al concepto representado $y$, relacionado (R), cuando el concepto identificado por el usuario es sinónimo directo o término que se identifica en el pictograma y compone el concepto esperado. Los porcentajes obtenidos en las pruebas se pueden observar en las tablas 1 y 2 . 


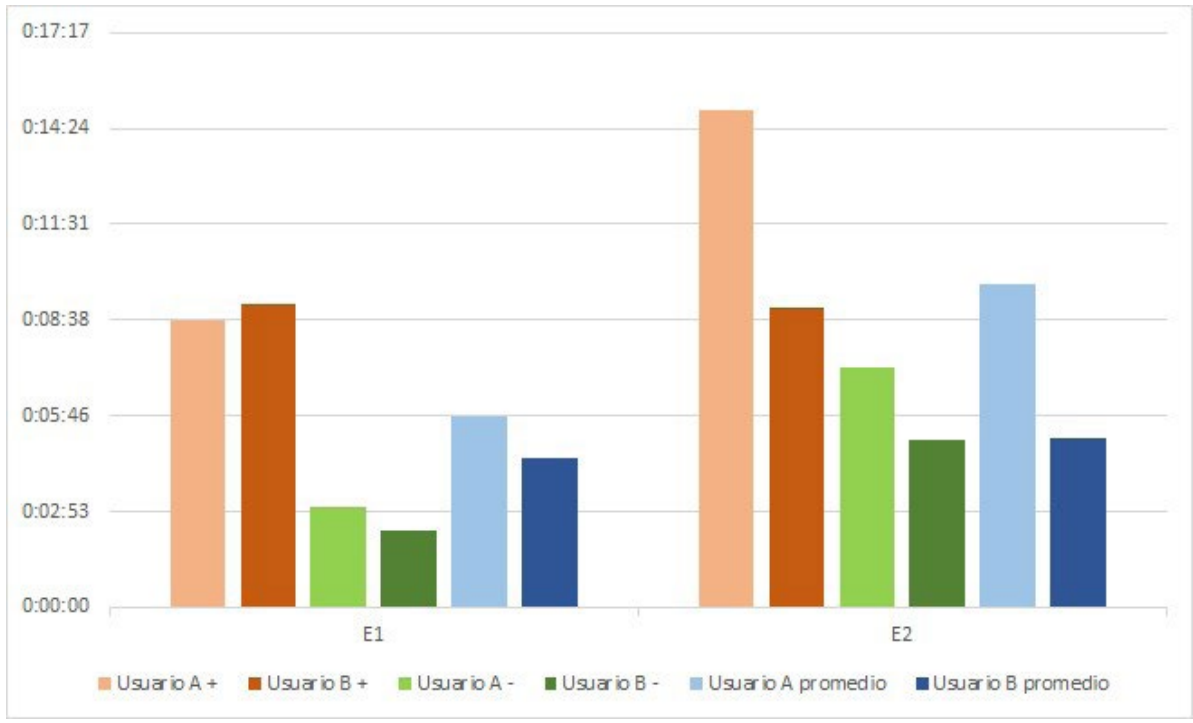

Figura 4. Tiempo máximo y mínimo por cada grupo de usuarios en las dos etapas (+ el que más tiempo tomó, - el que menos tiempo tomó).

\begin{tabular}{l|lll|lll|}
\cline { 6 - 7 } $\begin{array}{l}\text { Usuarios } \\
\text { A }\end{array}$ & $\mathrm{E} 1$ & & & $\mathrm{E} 2$ & \\
\cline { 6 - 8 } & $\mathrm{X}$ & $\mathrm{R}$ & $\mathrm{A}$ & $\mathrm{X}$ & $\mathrm{R}$ & $\mathrm{A}$ \\
\hline 1 & $62,50 \%$ & $25,0 \%$ & $12,50 \%$ & $63,7 \%$ & $0,0 \%$ & $36,3 \%$ \\
2 & $37,50 \%$ & $37,5 \%$ & $25,00 \%$ & $63,7 \%$ & $0,0 \%$ & $36,3 \%$ \\
3 & $62,50 \%$ & $25,0 \%$ & $12,50 \%$ & $63,7 \%$ & $0,0 \%$ & $36,3 \%$ \\
4 & $75 \%$ & $12,5 \%$ & $12,50 \%$ & $54,6 \%$ & $0,0 \%$ & $45,5 \%$ \\
5 & $54,54 \%$ & $0,0 \%$ & $45,46 \%$ & $9,1 \%$ & $63,6 \%$ & $27,3 \%$ \\
\hline Promedio & $58,41 \%$ & $20,00 \%$ & $21,59 \%$ & $50,9 \%$ & $12,7 \%$ & $36,3 \%$
\end{tabular}




\begin{tabular}{l|lll|lll|}
\multirow{2}{*}{$\begin{array}{l}\text { Usuarios } \\
\text { B }\end{array}$} & $\mathrm{E} 1$ & \multicolumn{5}{|l}{$\mathrm{E} 2$} \\
\cline { 2 - 7 } & $\mathrm{X}$ & $\mathrm{R}$ & $\mathrm{X}$ & $\mathrm{R}$ & $\mathrm{A}$ \\
\hline 1 & $50,00 \%$ & $25,00 \%$ & $25,00 \%$ & $27,27 \%$ & $27,27 \%$ & $45,45 \%$ \\
2 & $0,00 \%$ & $100,00 \%$ & $0,00 \%$ & $0,00 \%$ & $18,18 \%$ & $81,82 \%$ \\
3 & $25,00 \%$ & $62,50 \%$ & $12,50 \%$ & $0,00 \%$ & $36,36 \%$ & $63,64 \%$ \\
4 & $50,00 \%$ & $25,00 \%$ & $25,00 \%$ & $0,00 \%$ & $54,55 \%$ & $45,45 \%$ \\
5 & $37,50 \%$ & $37,50 \%$ & $25,00 \%$ & $0,00 \%$ & $63,64 \%$ & $36,36 \%$ \\
6 & $25,00 \%$ & $50,00 \%$ & $25,00 \%$ & $0,00 \%$ & $36,36 \%$ & $63,64 \%$ \\
7 & $37,50 \%$ & $37,50 \%$ & $25,00 \%$ & $45,45 \%$ & $9,09 \%$ & $45,45 \%$ \\
8 & $37,50 \%$ & $50,00 \%$ & $12,50 \%$ & $36,36 \%$ & $27,27 \%$ & $36,36 \%$ \\
9 & $50,00 \%$ & $50,00 \%$ & $0,00 \%$ & $0,00 \%$ & $36,36 \%$ & $63,64 \%$ \\
10 & $25,00 \%$ & $62,50 \%$ & $12,50 \%$ & $0,00 \%$ & $45,45 \%$ & $54,55 \%$ \\
11 & $37,50 \%$ & $50,00 \%$ & $12,50 \%$ & $18,18 \%$ & $36,36 \%$ & $45,45 \%$ \\
12 & $50,00 \%$ & $37,50 \%$ & $12,50 \%$ & $9,09 \%$ & $45,45 \%$ & $45,45 \%$ \\
\hline Promedio & $35,4 \%$ & $49,0 \%$ & $15,6 \%$ & $11,4 \%$ & $36,4 \%$ & $52,3 \%$
\end{tabular}

Consecuente a lo enunciado y observado, los individuos con NCC (usuarios A) superaron la cantidad de texto — términos - que se esperaba enunciaran en la E1, ellos no se limitaron a enunciar palabras, sino que realizaron un análisis de lo contenido en las imágenes, para referenciar y enunciar algunos términos que lee en los pictogramas. Lo anterior evidencia la tendencia a interpretar las relaciones existentes entre los componentes gráficos de los pictogramas, en última instancia analizan el contexto de cada una de las imágenes. La cantidad de porcentajes $(R)$ en los usuarios de tipo A demuestra la disposición que estos tienen para usar el material con el que cuentan en contexto en busca de aportar a su comunicación, lo anterior se ve más como un acto de apropiación y uso. Lo anterior permite entender que en la prueba el porcentaje de respuestas entre $R$ y A sean similares. El promedio de respuestas (R) $20 \%$ de la prueba, 
contrasta con el 58,4\% de no coincidencia o desacierto en la E1, la propuesta de pictogramas aumenta en su potencial de aporte a la comunicación, si se considera que los individuos la apropian y no solo hacen una lectura específica, estas dos respuestas $R$ y $A$ suman un margen superior al 65\% como acertada interpretación y posible recurso de comunicación.

Continuando con los individuos A, con respecto a la E2 se obtiene un porcentaje en aciertos (A) de 36,5\% y un 59,07\% de no aciertos (X) en la prueba. En E1 el $58,4 \%$ de pictogramas no fueron interpretados correctamente y en E2 el 50,9\% de pictogramas no fueron interpretados correctamente.

Los usuarios B evidenciaron en la prueba E2 un porcentaje de acierto A de $52,3 \%$ mientras que en la prueba E1 se obtuvo un acierto de tan solo $15,6 \%$. Los desaciertos en E1 fueron del 35,4\% y en E2 fueron de 11,4\%, que contrastan con la suma de aciertos y respuestas relacionadas para ambas pruebas que suman en E1 el 64,6\% y en E2 el 88,7\%, evidenciando una vez más que el acierto en interpretación como única respuesta acertada es desconocer que los individuos interpretan el contenido para usar la representación gráfica o pictograma. De nuevo se hace evidente la necesidad de acompañar las imágenes con texto y considerar el alto valor de su lectura en contexto.

El hecho de que en la prueba E1 los individuos B tengan más del 51\% de respuestas que relacionan términos con acierto y respuestas acertadas, indica que un pictograma se lee e interpreta para su uso, por lo que no puede ser absolutamente específico, al contrario, es un contenido flexible y puede representar conceptos o involucrar elementos que, en contexto del individuo, están relacionados con lo que quiere comunicar. En el caso de la E2 los aciertos y respuestas con términos relacionados suman un 88,7\% de interpretaciones como potencial de comunicación. 
A excepción de la prueba E1 para los usuarios A, que registraron un 41,59\% de respuestas acertadas y relacionadas con acierto, los demás porcentajes de interpretaciones realizadas por los usuarios A y B en las pruebas E1 y E2 superan porcentualmente los desaciertos en la lectura e interpretación.

A partir de los datos registrados en la ejecución de las pruebas, se evidencia que la presentación de contenido gráfico sin texto conlleva a una lectura e interpretación subjetiva y expresa generalmente más de un término dependiendo de los componentes presentes en el conjunto expuesto en el pictograma. La lectura se convierte en una fuente de comunicación en contexto dependiente de la experiencia que tiene el usuario, esto se concluye desde los términos y "descripciones" anotadas por los individuos partícipes en las pruebas. Lo anterior se considera como un potencial de comunicación para apropiarse y usarse en contexto, dado que de otra forma se consideraría que los pictogramas deben tener una única lectura, y en tal caso, el número de elementos gráficos que deberían ser presentados al usuario y los que éste debería seleccionar aumentan, proporcionalmente el proceso de búsqueda de los elementos se haría extenso, tedioso y en mucho complejo para los futuros usuarios.

De otro lado las pruebas permiten comprobar que el pictograma no pretende ser un elemento absolutamente específico, sino que por el contrario este se centra en una representación gráfica que busca expresar en un término que designa o enuncia una categoría de persona, situación, sentimiento, lugar, etc., pero que en contexto de uso y lectura por parte de cada individuo puede ser usada para relacionar y evocar aquello que no se puede hallar en otros pictogramas. Dado lo anterior, los contenidos gráficos que presenta el conjunto texto - pictograma, permiten una lectura y el eventual uso del contenido en diferentes contextos. También, los contenidos que tiene un pictograma pueden ser relevantes según el usuario los relaciona y por tanto, pueden ser usados para representar o relacionar diferentes conceptos. Es entonces pertinente, 
como parte de los nuevos procesos de los sistemas CAA, el entendimiento y apropiación en contexto de los contenidos de las imágenes para facilitar la producción de frases por parte de un usuario a partir de un conjunto mínimo de elementos.

Ya que parte fundamental de un sistema CAA son las frases resultantes del proceso de selección de pictogramas por el usuario, es relevante en el contexto de este artículo comprobar si el uso de una cantidad limitada de pictogramas permite crear frases con una estructura sintáctica de calidad. Para ello, se le pide al profesional en fonoaudiología (PF), quién conoce del proceso desarrollado para el diseño de los pictogramas y la interfaz gráfica de usuario, que basado en su conocimiento y experiencia en este tipo de casos, genere frases por medio de la selección de componentes gráficos. Se le pide al PF transcribir tres frases que diría una persona con afasia al momento de comunicar una actividad realizada durante el día. Luego se le pide que tome el rol de uno de los pacientes y seleccione los pictogramas necesarios para crear la frase, para lo anterior se debe tener en cuenta la capacidad de comunicación de la persona con afasia y el nivel de asociación que tiene con imágenes de este tipo. Finalmente, por medio de un sistema de generación de lenguaje natural, basado en la propuesta desarrollada por Narváez (Narváez et al, 2015)., se ingresan los conceptos que representa la imagen y se obtiene como resultado una frase.

En total se crean nueve frases correspondientes a tres pacientes, es importante mencionar que para la creación de frases el elemento mínimo debe ser una acción, si este es el caso, la frase estará en pasado y la persona realizará las acciones sin acompañantes. La selección de más componentes permite que las frases sean más completas. En la tabla 3 se presenta una muestra de los datos obtenidos de la prueba anterior y en la figura 5 se muestra un ejemplo de la selección realizada por el PF. 


\begin{tabular}{|l|l|l|}
\hline Frase sin ayuda tecnológica & Componentes seleccionados & Frase con ayuda tecnológica \\
\hline Fui allá a tomar chiquitico & $\begin{array}{l}\text { Lugar - parque } \\
\text { Persona - yo } \\
\text { Actividad - Tomar café }\end{array}$ & Tomé café en el parque. \\
\hline $\begin{array}{l}\text { Ayer allá película todos } \\
\text { bien }\end{array}$ & $\begin{array}{l}\text { Lugar - teatro de cine } \\
\text { Personas - yo/ Familia } \\
\text { Actividad - ir al cine } \\
\text { Tiempo- ayer } \\
\text { Motivo- gustar }\end{array}$ & $\begin{array}{l}\text { Ayer fui al cine con mi } \\
\text { familia porque me gusta. }\end{array}$ \\
\hline Hoy ella aquí bien tiempo & $\begin{array}{l}\text { Lugar - Casa } \\
\text { Personas - yo/amiga } \\
\text { Actividad - recibir visita } \\
\text { Emoción - felicidad }\end{array}$ & $\begin{array}{l}\text { Recibí visita en la casa con } \\
\text { mi amiga y me sentí feliz. }\end{array}$ \\
\hline
\end{tabular}

Tabla 3. Datos para la prueba de creación de frases.


Figura 5. Pictogramas seleccionados para la creación de la segunda frase.

Luego de obtener las frases, el PF contestó un cuestionario compuesto de ocho preguntas:

1. ¿Qué tanto se entendió el mensaje generado por el emisor con apoyo del programa?

2. ¿Fue completo el contenido de los mensajes generados?

3. ¿El mensaje logra ser lo suficientemente detallado?

4. ¿Los mensajes generados cumplen el propósito para el cual fueron generados por el emisor? 
5. ¿En qué grado el mensaje producido con la herramienta de comunicación concuerda con el mensaje que usted entendió al emisor?

6. Califique la calidad del mensaje producido con la herramienta de comunicación

7. ¿En qué grado el mensaje producido con la herramienta de comunicación concuerda con el mensaje que quería expresar el emisor?

8. ¿El mensaje generado corresponde a lo que el emisor deseaba expresar?

Las preguntas 1 a 7 tienen las opciones de respuesta muy bajo, bajo, alto, muy alto; y la pregunta 8 tiene las opciones sí y no. El 100\% de las frases generadas recibieron un sí en la pregunta 8 , lo que quiere decir que a la perspectiva del PF un emisor se sentiría satisfecho con la frase producida por medio del sistema. En la figura 6 se puede observar el nivel de satisfacción en cada una de las preguntas, en ningún caso una frase fue valorada como baja o muy baja lo cual valida el contenido generado y la capacidad de los pictogramas de representar conceptos de importancia para un usuario. En la pregunta número 1 se obtiene el mayor porcentaje de valoración muy alto con un 78\%, lo anterior indica que los mensajes generados por el sistema son entendibles y por tanto que tienen una estructura gramatical adecuada. Las preguntas 5 y 7 cuentan con el mismo porcentaje, lo anterior puede darse porque al ser el PF quien entiende en mayor medida a los usuarios con NCC es posible que encuentre una relación entre lo que entendió, que se esperaría sea lo cercano a la realidad, y lo que el usuario quería decir realmente. 


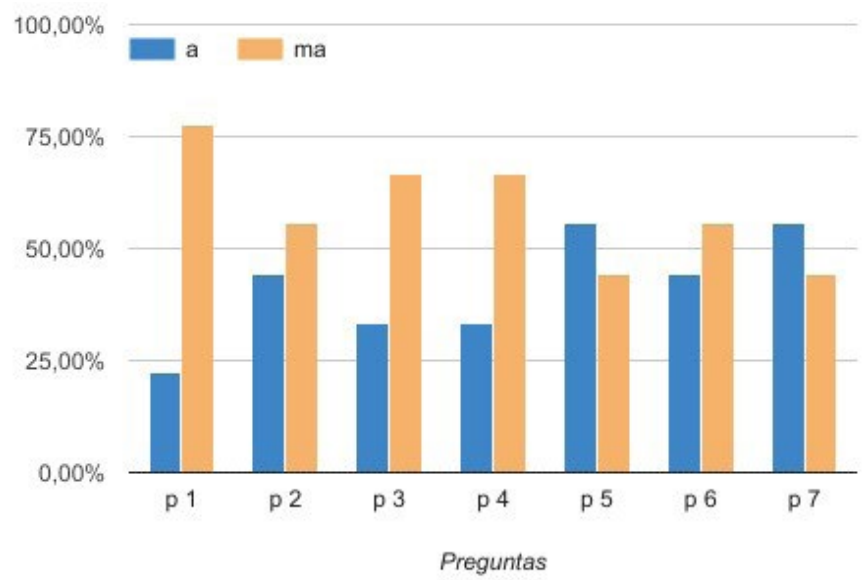

Figura 6. Porcentaje de satisfacción por pregunta en prueba de frases generadas por pictogramas.

Con lo anterior se confirma que la selección mínima de componentes gráficos como los pictogramas desarrollados en esta propuesta, que tienen en cuenta las expresiones verbales, el contexto del usuario y la cultura del mismo; permiten al usuario generar los mensajes que quieren decir por medio de un sistema computacional de comunicación.

\section{Conclusiones}

Los sistemas CAA han tomado gran importancia en la industria, lo que ha favorecido la creación de soluciones dinámicas y variadas; en este contexto muchas soluciones llevadas al mercado han generado interfaces gráficas que como tales repercuten en el manejo que hace el usuario de los sistemas y, desde este, la confianza y aprendizaje que derivan del uso de la aplicación. En este 
trabajo fueron identificados, por medio de una revisión en el estado del arte y una prueba de usabilidad, las características que debe tener la interfaz gráfica de un sistema CAA para su operación, además del cambio de la posición, tamaño y color de los elementos de la interfaz para su eficiente lectura y uso, se suma el uso de contenidos del contexto en el sistema.

Adicionalmente, se presenta una serie de pictogramas diseñados para su uso en un contexto local teniendo en cuenta la cultura y expresiones verbales que puedan tener los usuarios; las pruebas demuestran que para un usuario con NCC un pictograma puede representar diferentes conceptos, dado el contexto, lo anterior se entiende como una ventaja que facilita los procesos de interacción de los usuarios. Se comprueba la necesidad de incluir un término en los pictogramas que delimita las posibilidades de interpretación y uso por parte de las personas, facilitando el reconocimiento de los conceptos que se quieren usar en la composición de frases. Adicionalmente el uso de los pictogramas como ente facilitador de la interacción entre el usuario y el sistema se valida por medio de una prueba con un sistema de generación de lenguaje, encontrando que por medio de la selección de una cantidad mínima de componentes gráficos el usuario puede generar frases coherentes y eficientes por medio del sistema CAA.

Al considerar el estado del arte, la tecnología evaluada y la muestra seleccionada se logra experiencia y avance en el señalamiento de pautas para el diseño usable de interfaces apara usuarios con NCC. Entendiendo que estas características muestran una pauta de flexibilidad motora en la propuesta frente a las necesidades de los usuarios por su grado de familiaridad por su lenguaje gráfico y contexto. La propuesta se podría ampliar incluso a público que no tenga trastornos del lenguaje. 
Como trabajo futuro de esta propuesta se encuentra la validación de los componentes en conjunto del sistema CAA Relator, entre los cuales se encuentra el método de recomendación, el método de representación semántica, método de generación de lenguaje, interfaz gráfica personalizable y pictogramas para selección de conceptos.

\section{Agradecimientos}

Los autores de este trabajo quieren agradecer a los participantes de las pruebas y a Carolina Pardo, Carolina Rojas, Andrés González y Marcela Iregui; quienes aportaron en gran medida al desarrollo de nuestras ideas. Este trabajo fue desarrollado en el marco del proyecto INV ING-2103 financiado por la Vicerrectoría de investigaciones de la Universidad Militar Nueva Granada (UMNG) de la convocatoria 2016 y concluido en 2017.

\section{Referencias}

Al-Arifi, B., Al-Rubaian, A., Al-Ofisan, G., Al-Romi, N., \& Al-Wabil, A. (2013, July). Towards an arabic language augmentative and alternative communication application for autism. International Conference of Design, User Experience, and Usability,333-341. Springer, Berlin, Heidelberg. DOI:10.1007/978-3-642-39241-2_37.

Al Mahmud, A. and Martens, J. (2016). Social networking through email: studying email usage patterns of persons with aphasia. Aphasiology, vol. 30, 186-210.

Babic, J., Slivar, I., Car, Z. and Podobnik, V. (2015). Prototype-driven software development process for augmentative and alternative communication applications. 13th International Conference on Telecommunications (ConTEL). DOI:10.1109/ConTEL.2015.7231204. 
Belani, H. (2012, June). Towards a usability requirements taxonomy for mobile AAC services. Proceedings of the First International Workshop on Usability and Accessibility Focused Requirements Engineering (pp. 36-39). IEEE Press. DOI:10.1109/usare.2012.6226789.

Blake, M. (2000). Examining perceptions of graphic symbols across cultures: Preliminary study of the impact of culture/ethnicity. Augmentative and Alternative Communication, vol. 16, 180-185). DOI:10.1080/074346100 12331279034.

Davidson, B., Howe, T., Worrall, L., Hickson, L., and Togher, L. (2008). Social participation for older people with aphasia: The impact of communication disability on friendships. Topics in stroke rehabilitation, vol. 15, 325-340. DOI: $10.1310 /$ tsr1504-325.

Dumas, J. (2002, January). User-based evaluations. In The human-computer interaction handbook (pp. 1093-1117). L. Erlbaum Associates Inc. ISBN:08058-3838-4.

Higginbotham, D. and Bedrosian, J. (1995). Subject selection in AAC research: Decision points. Augmentative and Alternative Communication, vol. 11, 11-13. DOI:10.1080/07434619512331277099.

Hillis, A. (2007). Aphasia progress in the last quarter of a century. Neurology, vol. 69, 200-213. DOI: 10.1212/01.wnl.0000265600.69385.6f.

Hornero, G., Conde, D., Quílez, M., Domingo, S., Rodríguez, M., Romero, B. and Casas, O. (2015). A wireless augmentative and alternative communication system for people with speech disabilities., IEEE Access, vol. 3, 1288-1297. DOI:10.1109/access.2015.2466110.

International Organization for Standardization. (2018). Ergonomics of humansystem interaction — Part 11: Usability: Definitions and concepts (ISO 924111:2018). Recuperado de https://www.iso.org/obp/ui/\#iso:std:iso:9241:- 
$11:$ ed-2:v1:en.

Keskinen, T., Heimonen, T., Turunen, M. Rajaniemi, J. and Kauppinen, S. (2012). SymbolChat: A flexible picture-based communication platform for users with intellectual disabilities. Interacting with Computers, vol. 24, 374-386. DOI:10.1016/j.intcom.2012.06.003.

Lam, J. \& Wodchis, W. (2010). The relationship of 60 disease diagnoses and 15 conditions to preference-based health-related quality of life in Ontario hospital-based long-term care residents. Medical care, 48, 380-387. DOI: 10.1097/MLR.0b013e3181ca2647.

Light, J., \& McNaughton, D. (2012). The Changing Face of Augmentative and Alternative Communication: Past, Present, and Future Challenges. Augmentative and Alternative Communication, 28, 197-204. DOl: 10.3109/07434618.2012.737024.

Salminen, A., Petrie, H. and Ryan, S. (2004). Impact of computer augmented communication on the daily lives of speech-impaired children. Part I: Daily communication and activities., Technology and Disability, vol. 16, 157167. DOI:10.3233/tad-2004-16306.

Chan, R. (2014). Cloud augmentative and alternative communication for people with complex communication needs. Global Communications Conference (GLOBECOM) (2727-2732). IEEE. DOI:10.1109/glocom.2014.7037220.

Mancilla, D., Sastoque, S., \& Iregui, M. (2015, January). Towards ontology personalization to enrich social conversations on AAC systems. In 10th International Symposium on Medical Information Processing and Analysis (Vol. 9287, p. 92870Z). International Society for Optics and Photonics. DOI: $10.1117 / 12.2073865$.

Mancilla, D., Sastoque, S., Mendoza, J. and Iregui, M. (2013). Conceptualizing a daily activities ontology for an augmentative and alternative communication 
system. 5th Latin American Conference on Networked and Electronic Media (LACNEM-2013). Universidad Nacional de Colombia-Sede Manizales. DOI:10.1117/12.2073865.

Narváez, C. Sastoque, S. and Iregui, M. (2015). Spanish language generation engine to enhance the syntactic quality of AAC systems. 11th International Symposium on Medical Information Processing and Analysis (SIPAIM 2015). DOI:10.1117/12.2211435

Quesenbery, W. (2004). Balancing the 5Es of Usability. Cutter IT Journal, vol. 17, 4-11.

Hernández, S. S., Mancilla, D., Medina, J. M., \& Iregui, M. (2014). Usercentric Recommendation Model for AAC based on Multi-criteria Planning. International Conference on Advances in Human-oriented and Personalized Mechanisms, Technologies and Services. ISBN: 978-1-61208-369-8.

Sastoque, S., Narváez, C. y Garnica, G. (2016). Metodología para la construcción de Interfaces Gráficas Centradas en el Usuario. Nuevas Ideas en Informática Educativa, vol. 12, 314-324. ISBN: 978-956-19-0989-2.

Saturno, C., Ramirez, A., Conte, M., Farhat, M., y Piucco, E. (2015). An augmentative and alternative communication tool for children and adolescents with cerebral palsy. Behaviour \& Information Technology, vol. 34, 632-645. DOI:10.1080/0144929X.2015.1019567.

Seffah, A., Donyaee, M., Kline, R. y Padda, H. (2006). Usability measurement and metrics: A consolidated model. Software Quality Journal, vol. 14, 159178. DOI: 10.1007/s11219-006-7600-8.

Stent, A. \& Bangalore, S., Natural language generation in interactive systems. Cambridge University Press, 2014. DOI:10.1007/CBO9780511844492. 
The State of Queensland, Complex communication needs (2018). Retrieved from https://www.qld.gov.au/_data/assets/pdf_file/0022/58540/complexcommunication-needs.pdf

Van, E. and Alant, E. (2004). Associations with Minspeak ${ }^{\mathrm{TM}}$ icons. Journal of communication disorders, vol. 37, 255-274. DOl:10.1016/j. jcomdis.2003.10.002.

Como citar: Narváez, C.A. y Garnica, G.A. (2019). Lineamientos para el diseño de interfaces gráficas y componentes gráficos de sistemas de comunicación aumentativa y alternativa. Revista KEPES, 16 (20), 345375. DOI: 10.17151/kepes.2019.16.20.13 